\title{
Treatment of patients with spontaneous pneumothorax during videothoracoscopy
}

\author{
J.P. Janssen*, J. van Mourik**, M. Cuesta Valentin**, G. Sutedja*, \\ K. Gigengack ${ }^{\dagger}$, P.E. Postmus*
}

Treatment of patients with spontaneous pneumothorax during videothoracoscopy. J.P. Janssen, J. van Mourik, M. Cuesta Valentin, G. Sutedja, K. Gigengack, P.E. Postmus. CERS Journals Ltd 1994.

ABSTRACT: Thoracoscopy for spontaneous pneumothorax has been performed over the years by many pulmonologists. The aim of the procedure was merely diagnostic: the detection of blebs and bullae. Therapeutic modalities were restricted to chemical pleurodesis. The development of videothoracoscopy has made more complex interventions, such as bullectomy possible. A protocol for videothoracoscopic treatment of spontaneous pneumothorax, with all treatment modalities in one session, has been developed.

All patients with spontaneous pneumothorax underwent videothoracoscopy under general anaesthesia with double lumen tube intubation. If no abnormalities were found on the visceral pleura, talc pleurodesis was performed. Small lesions, blebs or bullae $<2 \mathrm{~cm}$, were coagulated prior to pleurodesis. In case of blebs or bullae $>2 \mathrm{~cm}$, thoracoscopic resection with an EndoGIA stapling device was performed, followed by scarification, i.e. electrocoagulation, of the parietal pleura.

In 43 patients, 44 procedures were performed. In 15 cases $(34 \%)$ no blebs or bullae were found. In 6 cases $(14 \%)$ only blebs $<2 \mathrm{~cm}$ were found. In 23 cases (52\%) blebs and bullae $>2 \mathrm{~cm}$ were found. In 21 out of 44 cases (48\%), talc pleurodesis was performed, and in 23 cases $(52 \%)$ bullectomy was performed. No major complication occurred. The average hospital stay was 5.7 days after talc pleurodesis and 6.0 days after bullectomy. There were 2 recurrences $(5 \%)$ after a follow-up of at least 18 months.

In conclusion, the use of videothoracoscopy in spontaneous pneumothorax makes it possible to continue a diagnostic procedure as a therapeutic session. The recurrence rate is low after both talc pleurodesis and bullectomy, whilst the hospital stay after the procedure is no longer than after chest tube drainage alone. Videothoracoscopic treatment may significantly reduce the need for thoracotomy in patients with spontaneous pneumothorax.

Eur Respir J., 1994, 7, 1281-1284.
Depts of * Pulmonary Diseases, ** Surgery and ${ }^{\dagger}$ Anaesthesiology, Free University Hospital, Amsterdam, The Netherlands.

Correspondence: P.E. Postmus

Dept of Pulmonary Diseases

Free University Hospital

De Boelelaan 1117

1081 HV Amsterdam

The Netherlands

Keywords: pleurodesis

spontaneous pneumothorax

videothoracoscopy

Received: November 31993

Accepted after revision April 31994
Spontaneous pneumothorax (SP) is a disease with a worldwide incidence of 5-15 patients per 100,000 persons a year [1]. It occurs 4-6 times more often in men than in women, with a peak incidence in adolescents.

There is no consensus on the treatment of SP. Treatment varies from "wait and see" policy to operative. The role of pleurodesis has not definitely been established [2]. If pleurodesis is not performed, the recurrence rate is between 20 and $60 \%[3,4]$.

More consensus exists on the treatment of recurrent SP. Patients with relapse are usually treated with pleurectomy and, in cases of bullae, bullectomy. This bullectomy is performed with the assumption of a causal relationship between the pneumothorax and the detected bullae.

The high recurrence rate of SP has prompted several investigators to perform traditional thoracoscopy in all cases of SP, to identify the cause [5-7]. In cases of blebs or bullae ("lesions") with a diameter less than 2 $\mathrm{cm}$, chemical pleurodesis was performed using talc or tetracycline. In cases of lesions with a diameter of more than $2 \mathrm{~cm}$, thoracotomy, bullectomy and pleurectomy were performed. Both pleurectomy and bullectomy and talc insufflation significantly reduce the recurrence rate of pneumothorax [2, 4, 5, 7]. Recent development of endoscopic stapler devices and video equipment has made it possible to perform interventional procedures under thoracoscopic guidance, which were traditionally reserved for thoracotomy $[8,9]$.

We developed a protocol (fig. 1) to incorporate surgical procedures in traditional thoracoscopy in SP. In addition to talc poudrage, in cases of lesions $<2 \mathrm{~cm}$, the lesions were coagulated. In case of lesions $>2 \mathrm{~cm}$, thoracoscopic resection was performed instead of thoracotomy, followed by scarification of the pleura. This article describes the first results of this approach. A further aim of the study was to establish the recurrence rate when using this protocol. 


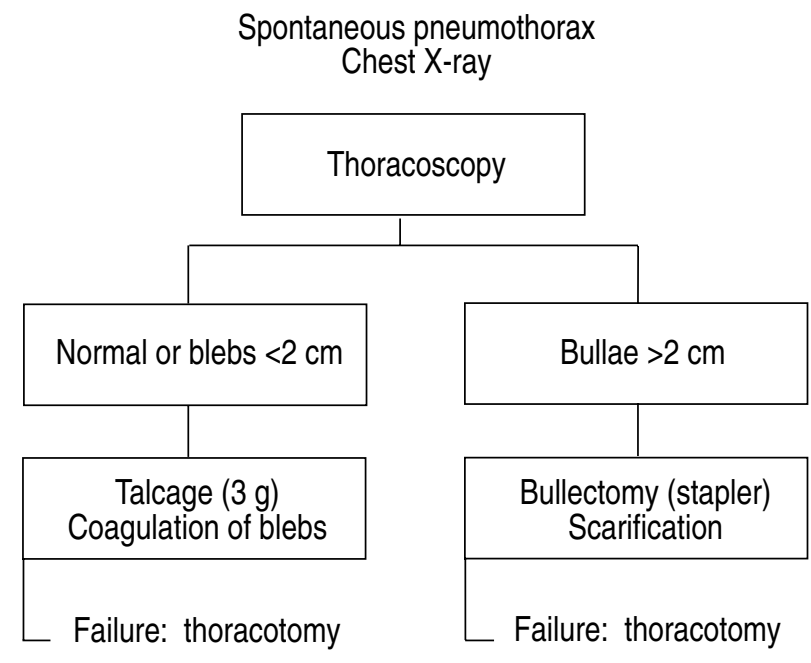

Fig 1. - Management protocol for patients with spontaneous pneumothorax.

\section{Material and methods}

\section{Patients}

Forty three patients with 44 events of SP were hospitalized. One patient was treated for SP on both sides, with only a one month interval. There were 32 men, age range $17-82 \mathrm{yrs}$, mean age $34 \mathrm{yrs}$, and 11 women, age range $20-51$ yrs, mean age 28 yrs. Twenty seven patients $(63 \%)$ were smokers, $4(9 \%)$ were ex-smokers. For 27 patients $(61 \%)$ this was the first event of pneumothorax. In 11 cases $(25 \%)$ it was the first (radiologically proven) ipsilateral recurrence. The first pneumothorax had been treated with bed rest ( 5 cases), drainage ( 3 cases), and thoracoscopy followed by pleurodesis (1 case). Treatment remained unknown in two cases. In 6 cases $(14 \%)$ it was the second or third recurrence. Four of these had also had a pneumothorax on the contralateral side $(1,19,25$ and 36 months earlier). Treatment consisted of bed rest, drainage, pleurodesis and, in one case, thoracotomy for a contralateral pneumothorax. In 25 cases $(57 \%)$ the pneumothorax was on the left side. Between December 1991 and August 1992, all patients presenting with a spontaneous pneumothorax were included in this protocol. Patients with a pneumothorax due to underlying pulmonary disease were excluded.

\section{Treatment protocol and definitions}

The protocol is presented in figure 1. Blebs are defined as small translucent vesicles with a thin nonvascularized wall; whereas, bullae have a vascularized surface, with a thick and usually opaque wall. Bullae are usually larger than blebs, and not as clearly demarcated from the lung $[10,11]$.

\section{Videothoracoscopy procedure}

Videothoracoscopy is performed in the operating theatre. The patient is under general anaesthesia, selectively intubated with a double lumen tube and placed in the lateral decubitus position with healthy side down. As is common for a normal thoracoscopy, the procedure is performed by a pulmonologist. If there seems to be indication for bullectomy, a surgeon operates.

After ventilation of the affected lung has been blocked, a $7 \mathrm{~mm}$ trocar (Wolf model "Boutin", Knittlingen, Germany) is inserted in the 5th intercostal space in the mid-axillary line. A $0^{\circ}$ optical telescope (Wolf "Panaview", Knittlingen, Germany) is inserted and connected to a videocamera (Wolf), which makes all endoscopic manoeuvres visible on a videomonitor (Sony). Under visual control, a second $(5 \mathrm{~mm})$ trocar is inserted in the $3 \mathrm{rd}$ intercostal space, anterior axillary line. Through this trocar a blunt instrument is inserted, which allows the operator to manipulate the collapsed lung. In this way, all parts of the completely collapsed lung can be inspected.

If a (collapsed) bulla is detected, the collapsed lung is partially reinflated, in order to investigate the full extent of the bulla.

In case of bullae larger than $2 \mathrm{~cm}$, a third trocar (12 $\mathrm{mm}$, Ethicon) is inserted, and through this trocar a stapling device (Autosuture EndoGIA 3.0 Multifire) is introduced. All visible bullae are stapled and removed. Subsequently, scarification of the parietal pleura is performed by electrocauterization at the level of the first five ribs. Lesions smaller than $2 \mathrm{~cm}$ are coagulated. If endoscopic resection is not necessary, talc poudrage is performed by insufflating $3 \mathrm{~g}$ of talc.

At the end of the procedure, a 20 FG (French gauge) drain is inserted, and connected to an underwater seal suction with a negative pressure of $20 \mathrm{cmH}_{2} \mathrm{O}$. The suction is continued for at least 3 days, or until the air leakage has stopped. If a control chest roentgenogram shows no residual pneumothorax, the drain is clamped for about $24 \mathrm{~h}$ and roentgenologically controlled for relapse. If there is no collapse, the drain is removed and the following day the patient is discharged.

\section{Results}

During thoracoscopy, no blebs or bullae were seen in 15 cases $(34 \%)$. Only small blebs were seen in 6 cases (14\%). Bullae of more than $2 \mathrm{~cm}$ were seen in 23 cases $(52 \%)$. Both bullae and blebs were seen in 5 of these $(11 \%)$.

Talc poudrage was performed in 21 cases $(48 \%)$. This was preceded by electrocoagulation of blebs in $6(14 \%)$. Bullectomy and scarification of the parietal pleura were performed in 23 cases $(52 \%)$. In 5 of these, coagulation of blebs was performed during the same procedure. Adhesions were seen in 10 cases $(23 \%)$. They were cut by electrocauterization in 9 cases $(23 \%)$. In one patient, extensive adhesions were present, and full inspection was not possible.

Of 44 patients, 27 were treated for first pneumothorax $(61 \%)$. In 18 of these patients $(67 \%)$ blebs or bullae were found. Of 17 patients treated for recurrent pneumothorax (39\% of all patients) lesions were found in $11(65 \%)$. 
Mean duration of hospitalization, after thoracoscopy when talc pleurodesis was performed, was 5.7 days (range 5-11 days), and 6.0 days when bullectomy was performed (range 5-13 days).

All patients were seen 2, 6 and 12 months after the intervention. With a follow-up period of at least 18 months so far, only two patients $(5 \%)$ have had a recurrence of pneumothorax, 2 and 3 months after bullectomy was performed. In one of these patients, video-thoracoscopy had been hampered by malposition of the double lumen tube. Consequently, this patient underwent thoracotomy.

\section{Complications}

One complication occurred due to the malfunctioning of the stapling device. This caused some damage to the lung parenchyma, resulting in prolonged air leakage of 13 days in the postoperative period. Other complications were: prolonged leakage after bullectomy in 4 patients (7-13 days); prolonged leakage after talc pleurodesis in 2 patients (6-7 days); and partial recollapse of the lung after clamping of the drain in 4 patients (three times after talc pleurodesis, once after bullectomy). In one case it was not possible to obtain a complete collapse of the affected lung due to malpositioning of the double lumen tube.

\section{Discussion}

No consensus exists about treatment of spontaneous pneumothorax, and treatment varies from conservative to bilateral thoracotomy. Conservative treatment has a high recurrence rate, and thoracotomy is associated with significant morbidity. This is a continuing dilemma for the clinician, the more so because no single clinical variable predicting the chance of recurrence is known. Blebs and bullae are often held responsible for causing pneumothorax although this has never been proved, and, particularily in younger patients, it is unusual to find one that is leaking. However, most investigators consider bullectomy to be a rational part of the treatment of (recurrent) pneumothorax. To prevent recurrence of pneumothorax, either chemical pleurodesis or pleurectomy is performed.

The videothoracoscopic approach method meets both conditions, and has some major advantages over other forms of treatment. Firstly, detection of the supposed cause of SP and treatment are performed in one session. Secondly, surgical procedures can be performed without thoracotomy. Thirdly, painful talc poudrage is performed under general anaesthesia, which contributes to the patients' comfort.

In our study, once the tube was well positioned and the lung completely collapsed, it appeared to be easy to inspect all parts of the lung, even the apices, where most lesions can be expected, but which are hard to inspect under local anaesthesia or without selective intubation. In addition, in case of detection of a bulla, inflation of the completely collapsed lung yielded important information about its size. With selective intubation of the patient, inflation and deflation could be easily performed. Lesions with a diameter of more than $2 \mathrm{~cm}$ were found in $52 \%$ of cases. This is a high number compared to studies in which traditional thoracoscopy was performed, (table 1). In a study by OLSEN and ANDERSEN [6] of 483 patients, $19 \%$ of patients had lesions $>2 \mathrm{~cm}$. In a study of 622 patients by VAN DE BREKel et al. [5], lesions $>2$ $\mathrm{cm}$ were found in $40 \%$ of cases.

In Olsen's study, the recurrence rate was $16 \%$, and at subsequent thoracotomy lesions larger than $2 \mathrm{~cm}$ were detected in 42 patients $(69 \%)$. In these patients, bullae were missed during the previous thoracoscopy, or had

Table 1. - Findings of bullous lesions during thoracoscopy: review of the literature considering traditional thoracoscopy versus findings in this study

\begin{tabular}{|c|c|c|c|c|c|c|c|c|}
\hline $\begin{array}{l}\text { First } \\
\text { author } \\
\text { [Ref.] }\end{array}$ & Year & $\begin{array}{c}\text { Patients } \\
\mathrm{n}\end{array}$ & $\begin{array}{c}\text { Lesions } \\
<2 \mathrm{~cm} \\
\%\end{array}$ & & $\begin{array}{c}\text { Lesions } \\
>2 \mathrm{~cm} \\
\%\end{array}$ & $\begin{array}{c}\text { Total } \\
\% \\
\end{array}$ & Treatment & $\begin{array}{c}\text { Recurrence } \\
\text { rate } \\
\%\end{array}$ \\
\hline \multicolumn{9}{|c|}{ Traditional thoracoscopy } \\
\hline $\begin{array}{l}\text { SwIERENGA } \\
\text { [3] }\end{array}$ & 1974 & 136 & 32 & & 17 & 49 & A & 7.7 \\
\hline $\begin{array}{c}\text { GUÉRIN } \\
\text { [4] }\end{array}$ & 1985 & 109 & 13 & & 41 & 57 & B & 5 \\
\hline $\begin{array}{c}\text { ALMIND } \\
{[14]}\end{array}$ & 1989 & 85 & $\longmapsto$ & $14 *$ & $\longrightarrow$ & 14 & B & 8 \\
\hline $\begin{array}{c}\text { Boutin } \\
\text { [7] }\end{array}$ & 1991 & 100 & 41 & & 17 & 58 & A & $* *$ \\
\hline $\begin{array}{c}\text { OLSEN } \\
{[6]}\end{array}$ & 1992 & 483 & $* *$ & & 19 & $* *$ & $\mathrm{C}$ & 16 \\
\hline $\begin{array}{l}\text { VAN DE BREKEL } \\
{[5]}\end{array}$ & 1993 & 622 & 15 & & 40 & 55 & A & 12 \\
\hline \multicolumn{9}{|c|}{ Videothoracoscopy } \\
\hline Our study & 1993 & 44 & 14 & & 52 & 66 & $\mathrm{D}$ & 5 \\
\hline
\end{tabular}

*: size of the lesions not differentiated; **: not mentioned. A: talc pleurodesis in case of lesions $<2 \mathrm{~cm}$, and thoracotomy in case of lesions $>2 \mathrm{~cm}$; B; talc pleurodesis in all patients (part of a comparative study); C: tetracycline pleurodesis in case of lesions $<2 \mathrm{~cm}$, and thoracotomy in case of lesions $>2 \mathrm{~cm}$; D: see text. 
occurred in the time between the thoracoscopy and thoracotomy. Sixty seven percent of recurrences occurred within 3 months after thoracoscopy (after 12 months of follow-up, two recurrences $(5 \%)$ were found in our group of patients). In Olsen's study, thoracoscopy was performed under general anaesthesia. In the study by VAN DE BREKEL [5], thoracoscopy was performed under local anaesthesia. In 20 out of 37 patients (57\%) who underwent thoracotomy for recurrence, bullous lesions larger than $2 \mathrm{~cm}$ were found. The lack of selective intubation of the patients in the latter study may explain the number of lesions missed during thoracoscopy. These studies were performed much earlier (OLSEN and ANDERSEN [6] 1979-1988; VAN DE BREKel et al. [5] 1965-1990). In recent years, however, not only have videomonitoring systems been introduced in thoracoscopy, but also light sources and optical telescopes have been significantly improved presumably resulting in better detection of blebs. Two patients in our group (5\%) suffered from recurrence after thoracoscopic bullectomy. In one of the two patients in our study who had a recurrent pneumothorax after videothoracoscopy, we had missed a bulla, as detected during thoracotomy. The videothoracoscopy in this patient, which was performed two months earlier, had been severely hampered by incomplete deflation of the affected lung, due to malposition of the double lumen tube. This case taught us that a correctly positioned double lumen tube and, consequently, a completely collapsed lung are indispensable for adequate inspection of the visceral pleura.

In the other patient with recurrent pneumothorax after videothoracoscopy, part of the bulla appeared to have been left underneath the suture line. At thoracoscopy, 3 months earlier, the size of the bulla had obviously been misjudged. Since then, we have performed a computed tomographic (CT) scan of the thorax before videothoracoscopy, to obtain a better assessment of the size and location of bullous lesions.

If we can avoid these mistakes in the future, we expect the recurrence rate to be further reduced. For patients with recurrent SP the approach described is probably preferable to thoracotomy.

It is unclear whether videothoracoscopic resection of bullous lesions without extensive efforts to obtain pleurodesis will be sufficient to prevent recurrence. In one study, this seemed to be true for bullectomy during thoracotomy [12]. For videothoracoscopic resection of bullae, a procedure which is less traumatic to the pleura, this might not be the case. Therefore, limited pleurodesis by scarification of the upper part of the parietal pleura close to the part of the lung where the lesions considered responsible for the pneumothorax are found, was added to the procedure. If no important abnormalities are found during videothoracoscopy, a limited pleurodesis is probably not sufficient. In these cases, chemical pleurodesis with talc should be performed, because of its good results in previous studies [13, 14].
As an initial treatment, videothoracoscopy is a more invasive treatment than bed rest or simple drainage. However, this procedure will considerably reduce the need for a second intervention as definitive treatment, compared to other initial treatments. Also, the hospitalization period is short. In our study, postoperative suction was continued for at least 3 days, to ensure complete and sustained re-expansion of the lung, which is necessary for successful pleurodesis. Whether the length of the suction period after talc can be reduced, is still a matter of discussion.

A randomized study, comparing videothoracoscopy with simple drainage as initial treatment and thoracotomy for recurrent disease, will be necessary to evaluate the possible benefit of this new procedure in terms of hospital stay, costs, and recurrence rate.

\section{References}

1. Neal JF, Vargas G, Smith DE, Akl BF, Sterling Edwards W. Bilateral bleb excision through median sternotomy. Am J Surg 1979; 138: 794-797.

2. Kirby TJ, Ginsberg RJ. Management of the pneumothorax and barotrauma. In: Buchalter SE, McElvein RB, eds. Clinics in Chest Medicine. Philadelphia, WB Saunders 1992; vol. 13, no. 1.

3. Swierenga J, Wagenaar JPM, Bergstein PGM. The value of thoracoscopy in the diagnosis and treatment of diseases affecting the pleura and lung. Pneumonologie 1974; 151: 11-18.

4. Guerin JC, Boniface E. Les methodes de pleurodese. Rev Prat 1990; 40: 1854-1856.

5. Brekel van de JA, Duurkens VAM, Vanderschueren RGJRA. Pneumothorax. Results of thoracoscopy and pleurodesis with talc poudrage and thoracotomy. Chest 1993; 103: 345-347.

6. Olsen PS, Orbaeck Andersen H. Long-term results after tetracycline pleurodesis in spontaneous pneumothorax. Ann Thorac Surg 1992; 53: 1015-1017.

7. Boutin C, Viallat JR, Aelony Y. Practical thoracoscopy. Berlin, Springer Verlag, 1991.

8. Bagnato VJ. Treatment of recurrent spontaneous pneumothorax. Surg Laparoscopy Endoscopy 1992; 2: 100103.

9. Coltharp WH, Arnold JH, Alford WC et al. Videothoracoscopy: improved technique and expanded indications. Ann Thorac Surg 1992; 53: 776-779.

10. Ohata M, Suzuki H. Pathogenesis of spontaneous pneumothorax. Chest 1980; 77: 771-776.

11. Miller WS. The Lung. 2nd edn. Springfield, Ill, CC Thomas, 1947.

12. Ferguson LJ, Imrie CW, Hutchinson J. Excision of bullae without pleurectomy in patients with spontaneous pneumothorax. Br J Surg 1981; 68: 214-216.

13. Guérin JC, Chambel F, Biron E, Kalb JC. Talcage pleural par thoracoscopie dans le traitement du pneumothorax. Rev Mal Respir 1985; 2: 25-29.

14. Almind M, Lange P, Viskum K. Spontaneous pneumothorax: comparison of simple drainage, talc pleurodesis, and tetracycline pleurodesis. Thorax 1989; 44: 627-630. 